Sección Sur-Sur

\title{
Análisis documental sobre el bullying utilizando la cartografía conceptual desde la so- cioformación
}

Documentary analysis of bullying using conceptual mapping from socioformation

\author{
Odete Serna Huesca | Escuela Normal Superior de México - MÉXICO | \\ Doctora en Pedagogía. Profesora Investigadora de Educación Superior en Escuela Normal Superior de México. Responsable del Cuerpo Académico en consolidación \\ "La Formación docente para la educación intercultural". E-mail: odetesh@hotmail.com
}

RESUMEN: El presente artículo reporta una investigación documental sobre el bullying haciendo énfasis en el enfoque socioformativo. Los propósitos son definir y diferenciar el bullying de otras violencias, describir una propuesta basada en la socioformación que contribuya a la transformación para promover una sociedad del conocimiento e identificar algunos elementos metodológicos que permitan evaluar a los docentes con base en la colaboración. Se utilizaron para el análisis documental, los ejes de la cartografía conceptual como una de las herramientas básicas de la socioformación. Las conclusiones señalan al bullying como un problema social presente en la escuela pero sus causas y consecuencias se extienden a otros ámbitos; en la socioformación no es necesario que este tipo de violencia escolar se presente de forma continua para considerarlo como tal por lo que las acciones que se planteen para su solución deben involucrar a todos los actores educativos y no solamente a los estudiantes.

Palabras clave: sociedad del conocimiento, violencia, socioformación, bullying

ABSTRACT: This article reports a documentary investigation on bullying emphasizing the socioformative approach. The purposes are to define and differentiate bullying from other violence, describe a proposal based on socioformation that contributes to the transformation to promote a knowledge society and identify some methodological elements that allow teachers to be evaluated based on collaboration. The axes of conceptual cartography were used for documentary analysis as one of the basic tools of socioformation. The conclusions point to bullying as a social problem present in the school but its causes and consequences extend to other areas; in socioformation it is not necessary that this type of school violence is presented continuously to consider it as such, so that the actions that are proposed for its solution must involve all educational actors and not only students.

Keywords: knowledge society, violence, socioformation, bullying 
La sociedad actual enfrenta retos diversos derivados de problemas relacionados con la violencia por lo que se requiere que en los diversos contextos en los que se desarrollan las personas, se promueva una sana convivencia en la que se valore la riqueza de saberes de los otros y la necesidad de promover relaciones de interdependencia positiva (Hamodi y Jiménez, 2018). Dichas actitudes y valores requieren ser modelados en los contextos familiar, escolar y social; sin embargo esto no siempre es así, ocasionando diversos problemas que se van acrecentando y deben ser atendidos por todos los actores educativos. Como ciudadanos y por supuesto, como miembros de una comunidad escolar debemos enfocarnos en una mejora de la calidad de vida a partir de la promover relaciones positivas buscando siempre la resolución de problemas de manera colaborativa (Tobón, Martínez, Valdez, y Quiriz, 2018); cocreando el conocimiento a partir de la indagación en fuentes confiables y rigurosas; promoviendo el pensamiento crítico y creativo en la búsqueda de una mejor calidad de vida con base en el tejido social, el emprendimiento y la convivencia a partir de acciones concretas que promuevan la sostenibilidad ambiental (Tobón, 2017a, 2017b).

El bullying es un problema que se ha intensificado en los últimos años en nuestro país y está afectando cada vez más a los estudiantes de todos los niveles educativos, con consecuencias diversas que incluso pueden llevar a las víctimas a la muerte (García, 2017). Este problema ha sido estudiado por distintos profesionales e investigadores desde distintas perspectivas, sin embargo, muchos de estos trabajos investigativos se han centrado en el diagnóstico para conocer si en los centros educativos existe o no el bullying, si los estudiantes son agresores, víctimas o espectadores, en alguno de los factores que tienen influencia en los comportamientos de los involucrados: familia, docentes, condiciones psicológicas o neuronales que pueden tener influencia en los comportamientos del bullying o bien, el diseño y/o aplicación de algunos programas de intervención emergentes para tratar de disminuir el problema (Garaigordobil, 2016; Garaigordobil et al., 2017; Valdés, Carlos, Wendlandt y Ramírez, 2016), sin embargo, poco se ha avanzado en planteamientos holísticos que incidan no solamente en los actores directamente involucrados (estudiantes) sino en los demás miembros tanto de la comunidad escolar como del entorno inmediato, que propicien un cambio en la realidad que impacte en la calidad de vida de todos (Tobón, 2017a, 2017b) .

Indudablemente, la escuela es un espacio fundamental para promover relaciones positivas que permitan un trabajo colaborativo en ambientes donde se practiquen valores esenciales para construir la sociedad del conocimiento (Tobón, et al., 2018), el enfoque socioformativo, que se encuentra en permanente construcción, promueve el trabajo conjunto con los diversos actores escolares y de la comunidad para la solución de problemas mediante proyectos inter y transdisciplinarios, basados en la colaboración, el proyecto ético de vida, la creación conjunta de saberes con la visión de complejidad, para promover la metacognición y el emprendimiento (Ambrosio, 2018). Este enfoque coincide con diversos aspectos con el socioconstructivismo pero va más allá al pretender la transformación de la realidad para mejorar la calidad de vida desde la sostenibilidad. Esto implica generar procesos innovadores que los participantes utilicen los conocimientos, habilidades, actitudes, aptitudes, valores, etc., para trascender el espacio áulico y generar propuestas con compromiso social. Dicho enfoque plantea oportunidades para avanzar no solamente en el conocimiento sobre el bullying sino también sobre propuestas para reducir el problema en el entorno escolar, que por supuesto, repercutirán en los distintos ámbitos en los que se desenvuelven los estudiantes. En este sentido, la socioformación puede contribuir a la resolución para tener una mejor calidad de vida; Tobón et al., (2018) plantean algunas acciones generales sobre la resolución al bullying desde los proyectos formativos.

El bullying y su definición es una temática que cobra cada día mayor importancia en la investigación. Una revisión general sobre el empleo del término en ScienceDirect, muestra que durante el período de 2015 a 2019 el número de publicaciones científicas que emplearon el término fue de 7761, mientras que en el período de 20142010, el número fue de 4777, lo que significa un aumento del 42.31\%; en relación al período 2005-2009 que fue de 2534, el porcentaje de aumento es de 67.34\%. En Redalyc se publicaron 1627 trabajos entre el 2015 y 2019 , representando un aumento del 19.05\% respecto al periodo anterior, 2010-2014, en el cual hubo 1317 publicaciones.

Sin embargo, a pesar de que el concepto de bullying cada vez tiene mayor relevancia, como se puede apreciar por la cantidad de investigaciones señaladas, no siempre ha tenido el mismo significado ni ha sido visto desde la misma perspectiva por lo que resulta necesario hacer un análisis sobre las concepciones y cambios que ha sufrido en el tiempo y en la forma de investigarlo, así como en la relación que existe con la violencia, la discriminación y la exclusión entre otros; así como las diferencias que hay con otros conceptos tales como violencia y específicamente violencia escolar. El presente estudio busca alcanzar las siguientes metas: 1) definir el bullying tomando en cuenta la socioformación; 2) diferenciar el bullying de otros tipos de violencias; 3) describir una propuesta basada en la socioformación factible en la educación que contribuya a la transformación de los contextos escolar y familiar que promuevan una sociedad del conocimiento; y 4) identificar algunos elementos metodológicos para evaluar y contribuir a mejorar en los docentes con base en la colaboración.

\section{DESARROLLO}

\subsection{Noción de Bullying}

El bullying es un término inglés que se utiliza en español como sinónimo de acoso escolar entre iguales, fue utilizado por Dan Olweus en 1970 para nominar el problema que era muy frecuente en los estudiantes. Se han real- 
izado diversas definiciones sobre el bullying que van integrando algunos elementos derivados de las investigaciones; dos de las características del problema son la continuidad y la intención con la que se realiza Chulli, Cárdenas, y Vilca, 2017; Lara-Ros, Rodríguez-Jiménez, Martínez-González, y Piqueras, 2017; Lugones, 2017; Hamodi y Jiménez, 2018; Abadio et al., 2018).

Desde la socioformación (Serna, 2019), el bullying es definido como un problema social en el que uno o varios estudiantes maltratan a otro u otros compañeros para causarles sufrimiento de distintas maneras (física, psicológica y/o social) sin necesidad de que se repitan las acciones de violencia para considerarlo como tal; esto se realiza con la permisividad de otros estudiantes y como producto de la interacción escolar, familiar y comunitaria; desde la socioformación es importante el abordaje de estos problemas mediante la gestión y cocreación del conocimiento, la articulación de diferentes saberes y el mejoramiento continuo en un entorno de inclusión, de tal manera que se contribuya a la sana convivencia de la sociedad.

\subsection{Categorización del bullying}

El bullying es una de las formas de violencia existentes en el ecosistema escolar, problema grave que forma parte de las causas del deterioro del ambiente social que es uno de los retos urgentes de asumir para crear condiciones propicias para transitar a la sociedad del conocimiento. El bullying es visto desde la socioformación como un problema que obstaculiza el proceso por el cual un estudiante apoya a otro para que se forme de manera integral mediante la resolución de problemas del contexto y la generación de productos relevantes, por medio de la retroalimentación continua. Implica promover la comunicación asertiva. Implica también la formación integral para promover mejores personas. Desde la socioformación, el bullying es un problema que compete a todos los actores educativos por lo que deben involucrarse para lograr el desarrollo social sostenible (Luna-Nemecio, 2020).

La visión socioformativa consiste en lograr que los estudiantes desarrollen competencias para la resolución de problemas de diferentes niveles de complejidad mediante el trabajo colaborativo con otros estudiantes, profesores, padres y en general, con la comunidad, considerando la construcción de conceptos, la creatividad y la articulación de diversos saberes. Implica la formación continua de los diversos actores educativos, especialmente los directivos y docentes (Tobón, 2017b). Es entonces, un proceso de formación multidireccional que involucra a todos los actores del proceso educativo.

Como se puede observar, no se trata de un problema simple que involucre solo a los estudiantes, sino a los miembros de la comunidad escolar y del entorno familiar del que son parte porque es un problema multifactorial que se manifiesta no solamente en aspectos comportamentales sino también fisiológicos, psicológicos y culturales entre otros (Cuevas y Marmolejo, 2016; Ruíz-Ramírez, Zapata-Martelo, García-Cué, Pérez-Olvera, Martínez-Corona y Rojo-Martínez, 2016). La socioformación involucra acciones colaborativas entre los miembros de la comunidad escolar con los actores del entorno de los estudiantes para realizar acciones integrales para la mejora social.

\subsection{Caracterización del bullying}

Tradicionalmente el bullying es considerado como un problema de difícil caracterización para los autores que, poco a poco, va siendo investigado más profundamente aunque se ha ido diversificando cada vez más con el uso de las TIC. Se visualizan en las investigaciones de diversos autores condiciones específicas para considerar y por supuesto, distinguir al bullying como tal: a) la temporalidad en la que se ejerce este tipo de violencia; b) la intención de causar daño a la o las víctimas; c) el abuso de poder del agresor que puede ocasionar sometimiento y otros graves daños en la persona sobre la que se ejerce el bullying (Esquivel, 2018; Cerezo y Rubio, 2017; Covarrubias y Caro, 2016; Garaigordobil, 2016).

En el bullying, autores como Cuevas et al (2016); Garaigordobil, Martínez-Valderrey y Machimbarrena (2017); Polo-Del Río, Felipe, León, Fajardo, Gómez y Mendo (2015) entre otros, están de acuerdo en que intervienen diversos actores: a) agresor o bully, b) víctimas pasivas o provocativas (Bonilla-Santos, González-Hernández y Bonilla-Santos, (2017); y c) observadores pasivos, activos y proactivos (Cuevas et al, 2016), aunque algunos de ellos han investigado más profundamente y han identificado variantes relacionadas con las conductas que se presentan.

Berger (2017) explica el origen del bullying a partir de tres modelos: 1) individuales, que señalan niños que disfrutarían agredir a otros, dominarlos, con poco desarrollo de habilidades emocionales, víctimas de bullying que los lleven a convertirse en agresores o incluso con características que pudieran ser genéticas; 2) modelos interpersonales y grupales, en los que se considera que entre los grupos existen jerarquías sociales y los estudiantes requieren adquirir status entre sus compañeros para ser aceptados por ellos; y 3) modelos socioculturales, en los que se parte de una serie de agresiones que históricamente se presentan en grupos contra personas vulnerables por alguna característica como raza, sexo, religión, etc.

El bullying es un problema complejo y multifactorial, entre las causas están las familiares, las educativas, las sociales, las culturales y las cognitivas, que se pueden presentar de forma conjunta y ocasionan violencias de distinta forma pero con resultados siempre negativos para las víctimas; sin embargo, los daños que genera el bullying afectan a los demás participantes aunque estos no lo consideren así. La naturalización de este problema rebasa el contexto 
áulico y se repite frecuentemente en otros contextos a través de distintas formas de violencia.

Sin embargo desde la socioformación, no es necesario que estas condiciones se presenten siempre sino que existan algunos de estos comportamientos. Para combatir este problema se plantean las siguientes características:

- Mejora de las relaciones entre los miembros de la comunidad escolar. La socioformación busca que los diversos actores educativos y comunidades mejoren sus condiciones de vida, identificando problemas importantes y generando soluciones factibles y pertinentes de ser implementadas en el entorno. Con ello, el aprendizaje no es la meta, sino un medio para tener la sociedad del conocimiento. -Colaboración de todos los actores educativos. Los proyectos formativos entendidos como un conjunto de actividades articuladas para la resolución de problemas del contexto; son estrategias para el fortalecimiento de programas formativos colaborativos que deben ser evaluados de forma concreta, para saber cuáles son los avances logrados en el desarrollo de las competencias (Hernández, Tobón, Ortega \& Ramírez, 2018).

- Pensamiento complejo. Como se ha planteado anteriormente, el problema del bullying no tiene una sola causa sino que es resultado de diversos factores por lo que se requiere de un análisis cuidadoso, crítico, creativo y sistémico para buscar soluciones con la participación de diferentes tipos de saberes que promuevan procesos para la mejora del entorno. La formación para la sociedad del conocimiento requiere de un pensamiento flexible que utilice de forma articulada, los conocimientos de distintas disciplinas o ámbitos de acción. El pensamiento complejo se integra por diversos principios como la dialógica, la recursividad, etc. (Morin, 1999).

- Metacognición. La metacognición, en términos generales se asume como el pensamiento sobre el propio pensamiento. En la socioformación, la metacognición se refiere al proceso continuo autovaloración que las personas realizan para mejorar su desempeño en el logro de metas, siempre acompañadas por valores universales como el respeto, la honestidad, la responsabilidad y la equidad entre otros (Tobón, 2017b). Como se puede observar, la metacognición rebasa la toma de conciencia sobre los procesos cognitivos realizados para aprender ya que también involucra los procesos afectivos y la actuación para el mejoramiento y la superación como seres humanos.

\subsection{Diferencias entre el Concepto de Bullying, violencia escolar y discriminación}

Muchas veces se utiliza el término violencia escolar como sinónimo de bullying aunque son problemas diferentes. La violencia escolar es una forma de violencia social en el que se manifiestan diversas formas de agresividad como la física, la psicológica, la sexual que puede ser realizada por cualquiera de los miembros de la institución escolar, es decir, abarca todas las acciones que causan perjuicio de cualquier índole a las personas, en el ámbito escolar. El bullying es un tipo de violencia escolar circunscrito al ámbito de los estudiantes en el que uno o más, agreden también, distintas maneras a otro u otros compañeros, en los que se observa un abuso de poder.

La discriminación se produce cuando una persona es tratada, por diversas características como el género, la raza, el origen étnico, una discapacidad, etc. de modo menos favorable que otra en una situación análoga a causa de su género, raza, etc., con lo que se viola su dignidad creándole un ambiente adverso (Rey, 2017)

El bullying está conformado por los siguientes características: 1) es una violencia ejercida de forma injustificada, por estudiantes contra otro u otros estudiantes; 2) puede trascender el contexto escolar y ejercerse en otros entornos como los virtuales; 3) existe una relación desigual del poder en el que el agresor abusa conscientemente de la víctima; 4) se realiza de manera continua por el agresor, casi siempre exhibiendo la violencia ante otros compañeros; 5) ocasiona siempre daño en las víctimas; y 6) es un problema multifactorial y complejo.

El bullying desde la socioformación difiere de la visión tradicional en los siguientes aspectos: 1) es un problema social que si bien se presenta entre estudiantes involucra a todos los actores educativos; 2) no es necesario que exista una acción continuada de agresión ya que es suficiente realizar algunos actos de sufrimiento con la intención de causar daño para que se dé esta denominación en esta perspectiva; y 3) los espectadores también desempeñan una función muy importante como elementos que pueden contribuir al problema por su inacción directa. Por lo anterior, es fundamental que se visualice al bullying como un problema que involucra a los integrantes del aula, de la escuela y de la comunidad; es uno de los retos actuales a vencer para combatir la violencia en muchos países del mundo.

\subsection{Tipos de actuación contra el bullying desde la Socioformación}

En la socioformación se proponen las siguientes prácticas para contribuir al combate del bullying: 1) motivación y apoyo para establecer relaciones de sana convivencia; 2) gestión del conocimiento y cocreación de saberes; 3) proyecto ético de vida; 4) trabajo colaborativo e inclusión; 5) comunicación asertiva; 6) transversalidad; 7) gestión de recursos; y 8) evaluación formativa metacognitiva. Se explicarán de manera general cada una de estas prácticas.

- Motivación y apoyo para establecer relaciones de sana convivencia. La motivación es lo que impulsa a una 
persona a realizar acciones de forma persistente para lograr sus objetivos manera sostenida: depende de factores externos e internos (Serrano, 2018). Desde la socioformación, es importante promover la motivación de los estudiantes para desarrollar sus competencias socioemocionales intrapersonales como la autoestima y la autoimagen así como las interpersonales en las que es fundamental la motivación externa para relacionarse con los demás de forma asertiva y empática. El docente tiene una función fundamental en la promoción de un clima favorable entre los estudiantes para que se practiquen actitudes y valores como el respeto, la empatía, la solidaridad y la colaboración entre otros. La intervención continua para promover la autorregulación de los alumnos es esencial.

- Gestión del conocimiento y cocreación de saberes. En este sentido se pretende que, en caso de que se presenten problemas como el bullying, el profesor aliente a los estudiantes en la búsqueda de soluciones de forma colaborativa. Para ello debe trabajar con todos los alumnos porque en algunos casos, pueden fungir como observadores pero se corre el riesgo de que se manifiesten posteriormente como agresores; todo lo anterior también promueve los valores universales esenciales en la sociedad del conocimiento, en la que se realiza la cocreación del saber de forma colectiva.

- Proyecto ético de vida. Esta práctica es fundamental para apoyar el desarrollo personal de los estudiantes con acciones que ayuden a formar mejores ciudadanos que no promuevan la violencia para lograr un tejido social pertinente para contar con condiciones de vida favorables para una comunidad sana en entornos de sostenibilidad ambiental con la presencia de los valores universales como responsabilidad, solidaridad, equidad, honestidad, respeto, promoción de la vida y compromiso entre otros (Tobón, 2017b). Los valores deben ser modelados no solamente en el aula sino en los distintos entornos en los que los estudiantes se desarrollan.

- Comunicación asertiva. Esta práctica es fundamental también para promover una interacción constante entre los alumnos para que aprendan a expresarse con otras personas de manera clara (indicando lo que se busca), cordial (amable) y respetuosa (sin agresiones verbales) mediante la escucha activa, con el fin de lograr una determinada meta, resolver un problema o tomar una decisión, siguiendo los valores universales. En la educación, todos los actores deben promover la práctica de este tipo de comunicación, y buscar que se fortalezca haciendo reflexiones sobre cómo nos comunicamos y podemos mejorar, a través del apoyo de unos con otros, lo cual incide en el mejoramiento del ambiente escolar.

- Trabajo colaborativo e inclusión. La resolución de un problema debería ser producto de acciones de todos los actores educativos posibles, para obtener mejores resultados que cuando solamente se realizan acciones individuales (Vázquez, Hernández, Vázquez, Juárez, y Guzmán, 2017). En el bullying se trata de lograr que los alumnos aprendan a convivir, respetar las diferencias, negociar, trabajar con los otros para resolver los conflictos que se presenten en los grupos para convivir sanamente. Todos los actores educativos deben realizar trabajo colaborativo de manera continua para fortalecer comunidades de aprendizaje.

- Transversalidad. La atención al problema del bullying no es responsabilidad solamente del docente que esté al frente del grupo en el momento en el que surja la violencia entre los estudiantes sino que están involucrados compañeros, docentes, autoridades, familiares y todas aquellas personas que estén presentes en la vida de los adolescentes, es decir, la formación se promueve en distintos ámbitos de acción: escolar, familiar y social; puede considerarse como una forma de afrontar los procesos educativos de manera más sistémica. Desde la socioformación, consiste en lograr que los estudiantes actúen saberes de varias áreas para identificar, analizar, explicar y resolver un determinado problema contextual desde otra perspectiva.

\subsection{Vinculación del bullying}

El bullying se vincula con el trabajo colaborativo entendido desde la socioformación como el proceso que realizan las personas, de forma conjunta, en el que comparten y articulan sus competencias para lograr una meta común, obteniendo un mayor impacto que si la tarea hubiera sido realizada por una sola persona (Tobón, 2017a). Dicho trabajo colaborativo, en el caso del bullying, debe estar presente en todos los contextos de acción en los que se presente el problema, es decir, en el aula, en la escuela, en la familia y de ser posible, en la comunidad en la que viven los alumnos a partir de las siguientes acciones: 1) acordar una meta concreta con la que se identifiquen todos; 2) diseñar e implementar un plan de acción conjunto; 3) realizar un trabajo en el que se complementen las acciones con las fortalezas de los integrantes para lograr mejores resultados; 4) realizar procesos metacognitivos continuos para reflexionar sobre los aciertos y errores con el propósitos de reorientar las acciones para el logro de la meta; 5) comunicarse asertivamente, promoviendo los valores esenciales en una sana convivencia y; 6) asumir con responsabilidad personal las actividades que hayan sido acordadas en el plan de acción por todos los participantes.

Como se puede observar, la vinculación existente entre bullying y trabajo colaborativo requiere de acciones en diversos sentidos de forma holística en distintos niveles de acción en virtud de la complejidad del problema por lo que es fundamental el compromiso de todos para lograr una mejor calidad de vida en la sociedad del conocimiento con un desarrollo social sustentable. El docente, en un primer momento, se asume como un líder en la intervención para combatir el problema, pero deben integrarse los demás miembros de los entornos en los que se desenvuelven 
los estudiantes porque las soluciones no son sencillas como las sanciones que en ocasiones, complejizan más el problema.

\subsection{Metodología para combatir el bullying desde la Socioformación}

La metodología que se propone, desde la socioformación, para solucionar o cuando menos reducir el problema del bullying, en este caso, está basada en los proyectos formativos para identificar, interpretar, argumentar y actuar sobre un problema del contexto para lograr el desarrollo social sostenible, en el que se obtiene un producto tangible o intangible, a partir de actividades articuladas de forma colaborativa, enmarcados en el pensamiento complejo y en la gestión del conocimiento obtenido en fuentes pertinentes, confiables y rigurosas (Tobón, 2017a).

Los proyectos formativos buscan comprender, argumentar y resolver problemas del contexto en ámbitos como el personal, familiar, social o ambiental entre otros, de manera integral, para beneficio de las personas. Inician de la búsqueda del conocimiento, organizándolo, analizándolo, comprendiéndolo, adaptándolo, creando, innovando y aplicando propuestas diversas mediante el trabajo colaborativo de forma incluyente enriqueciéndose de las diferencias existentes en las personas respetando la diversidad de creencias, capacidades, opiniones, etc., aprovechando sus fortalezas para el logro de las metas de manera más efectiva; además se busca el desarrollo de habilidades de pensamiento complejo en el marco de la gestión del conocimiento. forma flexible:

Los proyectos formativos se integran metodológicamente de las siguientes fases que son realizadas de

\section{a) Presentación del problema}

Para realizar estas acciones se requiere a) Identificar el problema que se desea resolver con los involucrados; b) Precisar el o los ámbitos de acción (personal, familiar, social) que están involucrados en el problema; c) Señalar el problema en forma de pregunta o de un sencillo enunciado entendible para todos; d) hacer explícita la vinculación existente entre las distintas áreas involucradas en la resolución del problema; e)motivar a todos para colaborar en la resolución del problema; establecer las acciones claves del proyecto de forma conjunta y; sistematizar las responsabilidades de cada participante en el proyecto.

\section{b) Análisis de saberes previos}

Esta actividad se puede realizar de formas diversas como la presentación de casos, un video, una noticia actual, lluvia de ideas, organizadores, etc., que permitan recabar información sobre lo que conocen los participantes sobre el bullying para identificar los aspectos que no son contemplados por ellos para realizar la búsqueda de información o materiales que propicien la reflexión sobre el impacto negativo, las consecuencias y daños que se pueden ocasionar si no se realiza una intervención conjunta de manera responsable.

\section{c) Gestión del conocimiento}

Con el propósito de conocer el problema de forma integral es importante a) la puesta en común del concepto o conceptos claves implicados en el problema para contar con elementos semejantes en todos los participantes; b) realizar una búsqueda de información sobre el bullying para posteriormente, analizarla, valorarla y utilizarla durante el proceso de implementación de los proyectos formativos; c) establecer acuerdos con los demás participantes para contar con un marco teórico común de saberes que propicien la resolución del problema y; d) adquirir argumentos confiables sobre el fenómeno del bullying que ayuden en el combate a la situación problemática.

\section{d) Diagnóstico del contexto}

Es fundamental a) conocer a profundidad el contexto en el que se pretende contribuir a la solución del problema; b) hacer un diagnóstico de manera puntual y precisa, de los aspectos más relevantes en los que se está presentando el problema ya que dicha revisión permitirá visibilizar elementos o factores que pueden propiciar situaciones que vuelvan más complejo el problema e incluso, lo agraven y para ello es necesario; c) buscar los recursos pertinentes y disponibles en el contexto que puedan utilizarse como herramientas para resolver el bullying.

\section{e) Emprendimiento de acciones para la resolución del problema en el contexto}

Esta fase implica un proceso metacognitivo interesante porque además de trabajar colaborativamente para diseñar acciones pertinentes al contexto diagnosticado a) considera los escenarios prospectivos deseables con respuestas acordes a las personas y recursos disponibles; b) propone opciones discutidas y consensuadas para el logro de la meta y; c) se aplican saberes de varias áreas o ámbitos para responder a la complejidad de causas que inciden en el problema del bullying. 


\section{f) Socialización de los productos logrados}

Una vez que se ha realizado el proyecto es fundamental realizar, entre otras, las siguientes actividades: a) presentación de las evidencias y productos que se fueron generando en el proceso de resolución del problema acorde con unos determinados criterios; b) coevaluación de dichas evidencias y productos para recibir retroalimentación de otros participantes para lograr un mejoramiento continuo; c) compartir los productos y experiencias obtenidos con otros estudiantes, directivos, familiares, miembros de la comunidad, etc., para socializar formas de resolver los problemas colaborativamente para obtener mejores resultados (Tobón, 2014).

\section{CONCLUSIONES}

El empleo del concepto de bullying está en aumento en la literatura científica, se tiene consenso en su definición pero no así en su naturaleza ni en los elementos que lo estructuran. En el presente análisis se pretendió avanzar en este aspecto realizando planteamientos diversos desde la socioformación; el bullying, desde este enfoque, es considerado como un problema social en el que uno o varios estudiantes acosan a otro u otros estudiantes para causarles sufrimiento de manera física, psicológica y/o social; en presencia o con el conocimiento de otros pares en el ámbito escolar pero puede extenderse a otros ámbitos.

Desde la socioformación no es necesario que se repitan las acciones de violencia para hablar de una situación de bullying porque un acto puede causar daños graves a la víctima. En esta perspectiva se plantean propuestas para promover la resolución del problema con acciones colaborativas propuestas e implementadas con los diversos actores educativos en un marco ético para la mejora de la calidad de vida de tal manera que esto contribuya al desarrollo social sostenible. El bullying no es un problema sencillo y debe ser pensado desde la complejidad promoviendo la metacognición. Los proyectos formativos pueden ser herramientas muy útiles para involucrar a las diversas comunidades involucradas.

El análisis conceptual aquí expuesto presenta de forma general, las aportaciones de algunas investigaciones, pero propone además algunas propuestas desde la socioformación de acuerdo con la sociedad del conocimiento y los retos del desarrollo social sostenible, con el propósito de ser analizadas, valoradas y discutidas por otros investigadores para fortalecerlas o mejorarlas a partir de diversas experiencias y saberes. Se trata de integrar en el centro del debate, estas propuestas para el desarrollo de este enfoque, en un área que se convierte en una demanda emergente por las consecuencias que tiene no solamente en los estudiantes sino en la sociedad en general. Este problema requiere del uso del pensamiento crítico, sistémico, creativo y flexible, por lo que se necesita profundizar en el estudio.

\section{REFERENCIAS BIBLIOGRÁFICAS}

Abadio, Wanderley; lossi, Marta Angélica; Carvalho, Flávia; Lopes, Denise; Mariano, Andréa Cristina \& Carvalho, Deborah. (2018) Causas del bullying: resultados de la Investigación Nacional de la Salud del Escolar. Revista Latino-Americana de Enfermagem, 23(2), 275-282.

Ambrosio, Ricardo. (2018). La socioformación: un enfoque de cambio educativo. Revista lberoamericana de Educación, $76(1), 57-82$.

Berger, Christian. (2017). Bullying, Ficha. Gobierno del Ministerio de Educación Chile: Convivencia Escolar, 2 - 5.

Bonilla-Santos, J., González-Hernández, A. \& Bonilla-Santos, G. (2017). Neuroendocrine characteristics of early trauma and its relation to bullying. Revista Ecuatoriana de Neurología, 26(3), 258-265.

Cerezo, F., \& Rubio, F. (2017). Medidas relativas al acoso escolar y ciberacoso en la normativa autonómica española. Un estudio comparativo. Revista Electrónica Interuniversitaria de Formación del Profesorado, 20(1), 113--126.

Chulli, Deysi \& Cárdenas, J. (2016) Funcionamiento familiar y bullying en estudiantes de instituciones educativas públicas del distrito de Lurigancho. Revista de Investigación Apuntes Psicológicos 2(1), 43-52.

Covarrubias, Arlette \& Caro, Nelly. (2016). Determinantes de la violencia entre pares en escuelas secundarias del Estado de México: calidad de relaciones con maestros y familiares. Revista lberoamericana la Investigación y el Desarrollo Educativo: RIDE, 7(13), p.12

Cuevas, María \& Marmolejo, María. (2016). Observadores: un rol determinante en el acoso escolar. Pensamiento Psicológico, 14(1) 89-102.

Esquivel, Daniela. (2018). Los prejuicios asociados a la hora de catalogar a los actores del bullying. Trabajo social, $17(1), 43$.

Garaigordobil, Maite. (2016). Conducta antisocial: conexión con bullying/cyberbullying y estrategias de resolución de conflictos. Psychosocial Intervention. 26(1), 47-54.

Garaigordobil, M., Martínez-Valderrey, V. \& Machimbarrena, J. (2017). Intervención en el bullying y cyberbullying: Evaluación del caso Martín. Revista de Psicología Clínica con Niños y Adolescentes, 4(1), 25-32.

García, Maritza \& Ascencio, Christian. (2015). Bullying y violencia escolar: diferencias, similitudes, actores, consecuencias y origen. Revista Intercontinental de Psicología y Educación, 17(2), 9-38.

García, Ricardo. (2017). Reflexión. Bullying a la verdad. Enseñanza e Investigación en Psicología, 22(2), 261 -262.

Hamodi, Carolina \& Jiménez, Leire. (2018). Modelos de prevención del bullying: ¿qué se puede hacer en educación infantil?. IE Revista de investigación educativa de la REDIECH, 9(16), 29-50. 
Hernández, J., Tobón, S., Ortega, M., \& Ramírez, A. (2018). Evaluación socioformativa en procesos de formación en línea mediante proyectos formativos. Educar, 54(1), 0147-163.

Lara-Ros, M., Rodríguez-Jiménez, T., Martínez-González, A., \& Piqueras, J. (2017). Relación entre el bullying y el estado emocional y social en niños de educación primaria. Revista de psicología clínica con niños y adolescentes, 4(1), 59-64.

Lugones, Miguel \& Ramírez, Marieta (2017). Bullying: aspectos históricos, culturales y sus consecuencias para la salud. Revista Cubana de Medicina General Integral, 33(1), 154-162.

Luna-Nemecio, J. (2020). Para pensar el desarrollo social sostenible: múltiples enfoques, un mismo objetivo. Mount Dora (USA)/Quito (Ecuador): Kresearch/ Religación. Centro de Investigaciones en Ciencias Sociales y Humanidades desde América Latina.

Morin, E. (1999). Introducción al pensamiento complejo. Barcelona: Gedisa.

Polo-Del Río, María. (2015). Análisis de la Socialización sobre Perfiles de la dinámica bullying. Universitas Psychologica. 14(3), 11171127.

Rey, Fernando (2017). Igualdad y prohibición de discriminación: de 1978 a 2018. Revista de Derecho Político, 1(100), $125-171$.

Vásquez, Johnny. (2018). Análisis sobre la motivación educativa. Revista Mapa, 2(8).

Ruíz-Ramírez, Rosalva; Zapata-Martelo, Emma; García-Cué, José; Pérez-Olvera, Antonia; Martínez-Corona, Beatriz \& Rojo-Martínez, Gustavo (2016). Bullying en una Universidad Agrícola del Estado de México. Ra Ximhai. 12(1), 105-126.

Serna, Odete. (2019). ). Cartografía Conceptual del Bullying: Hacia la Teorización e Intervención desde la Socioformación. Atenas, 3(47), 124-141.

Tobón, Sergio. (2014). Proyectos formativos: teoría y práctica. México: Pearson.

Tobón, Sergio. (2017a). Essential axes of knowledge society and socioformation. Mount Dora: Kresearch.

Tobón, Sergio. (2017b). Conceptual analysis of the socioformation according to the knowledge society. Knowledge Society and Quality of Life (KSOL), 1(1), 9-35

Tobón, Sergio; Martínez, Jorge; Valdez, Eliseo \& Quiriz, Tania (2018). Prácticas pedagógicas: Análisis mediante la cartografía conceptual. Revista Espacios, 39(53).

Tobón, Sergio; Guzmán, Clara; Pérez-Estrada, Rosalía \& Díaz-Azuara, Alfredo (2019). El Talento: Un Análisis Socioformativo. En Luis Gibrán, Juárez- Hernández: Josemanuel, Luna-Nemecio y Clara, Guzmán (Coords.), Talento, investigación y socioformación (pp. 17-35). Mount Dora (USA): Kresearch.

Valdés, Á., Carlos, E., Wendlandt, T., \& Ramírez, M. (2016). Propiedades psicométricas de una escala para medir el manejo de la vergüenza en adolescentes (moss-sast). Acta Colombiana de Psicología. 19(1), 24-34.

Vázquez, J., Hernández, J., Vázquez-Antonio, J., Juárez, Luis \& Guzmán, C. (2017). El trabajo colaborativo y la socioformación: un camino hacia el conocimiento complejo. Revista Educación y Humanismo, 19(33), 334-356.

How to cite this article: Serna Huesca, O. (2020). Análisis documental sobre el bullying utilizando la cartografía conceptual desde la socioformación. Religación. Revista De Ciencias Sociales Y Humanidades, 5(23), 155-162. Recuperado a partir de http://revista.religacion.com/index.php/religacion/article/view/609

Submitted: 13 January 2020 Accepted: 17 March 2020 Published: 31 March 2020

Religación. Revista de Ciencias Sociales y Humanidades is a peer-reviewed open access journal published by CICSH-AL Centro de Investigaciones en Ciencias Sociales y Humanidades desde América Latina |Religación| 